\title{
Development of a Process Assessment Model for assessing Medical IT Networks against IEC 80001-1
}

\author{
Silvana Togneri MacMahon ${ }^{1}$, Fergal Mc Caffery ${ }^{1}$, Sherman Eagles ${ }^{2}$, Frank \\ Keenan $^{1}$, Marion Lepmets ${ }^{3}$, Alain Renault ${ }^{3}$ \\ ${ }^{1}$ Regulated Software Research Group, Department of Computing \& \\ Mathematics, Dundalk Institute of Technology \& Lero, Dundalk Co. Louth, \\ Ireland \\ ${ }^{3}$ Public Research Centre Henri Tudor, Luxembourg \\ \{alain.renault, marion. lepmets\} @tudor.lu
}

\begin{abstract}
Increasingly medical devices are being designed to allow them to exchange information over an IT network. However incorporating a medical device into an IT network can introduce risks which can impact the safety, effectiveness and security of the medical device. Medical devices are stringently tested according to regulation during the design and manufacture process. However until the introduction of IEC 80001-1: Application of Risk Management for IT-Networks incorporating Medical Devices, no standard addressed the risks of incorporating a medical device into an IT network. In order to perform an assessment (which is compliant with ISO/IEC 15504-2) of an IT network against IEC 80001-1, a Process Assessment Model is required. Based on the relationship between IEC 80001-1 and ISO/IEC 20000-1, this paper examines how the TIPA transformation process developed by Public Research Centre Henri Tudor was used to develop a process assessment model (TIPA PAM) for ISO/IEC 20000-1. It also examines how a process assessment model can be developed following that transformation process to assess Medical IT networks against IEC 80001-1.
\end{abstract}

Keywords: IEC 80001-1, ISO/IEC 15504 - Process Assessment, Service Management, ISO/IEC 20000-1, TIPA, ITIL.

\section{Introduction}

As Medical Devices are increasingly being designed to be incorporated into hospital IT networks, IEC 80001-1 [1] addresses the risk that this involves. Risks to the safety, effectiveness and security of the system are addressed. These risks are examined in more detail in section 2. The design and production of medical devices is subject to regulation under various standards which are recognised by the regulatory 
authorities within the region in which the device will be marketed. The incorporation of a medical device into an IT network establishes a Medical IT network. Medical IT networks are increasingly being required to carry diverse traffic from confidential patient information to generic email traffic. Until the introduction of IEC 80001-1, no standard was in place to address the risk that health care can be compromised when a medical device is incorporated into an IT network. IEC 80001-1 seeks to address Life Cycle Risk Management in Medical IT networks and focuses on achieving interoperability of devices on the network without compromising safety, effectiveness or data and system security.

The next section of the paper examines why the IEC 80001-1 standard was developed by looking at the risks that are inherent in the incorporation of Medical Devices into IT networks. Section 3 provides a brief overview of how Process Assessment Model (PAM) may be developed to assess against this standard. In order to develop this assessment model our research has investigated the relationship between IEC 80001-1 and ISO/IEC 20000-1 - Information Technology - Service Management - Part 1: Service management system requirements [2]. Prior to the introduction of IEC 80001-1, ISO/IEC 20000-1 had been published as a generic Service Management Standard. ISO/IEC 20000-1 provides requirements for a service provider to deliver managed service throughout the life cycle. IEC 80001-1 recognises the need for a life cycle approach to risk management of the incorporation of medical devices onto IT networks. As such IEC 80001-1 is similar to ISO/IEC 20000-1, focusing on the specific risks inherent in the Service Management of medical devices within an IT network. Section 4 examines the requirements for process assessment that are defined in ISO/IEC 15004-2 (Process Assessment - Part 2: Performing an assessment). Section 5 describes ISO/IEC 20000 (Parts 1, 2 and 4) while Section 6 examines the relationship between IEC 80001-1 and ISO/IEC 20000 (Parts 1 and 2). On the basis of this relationship our research investigated methodologies that have been developed to assess against ISO/IEC 20000-1 processes. This paper focuses on the TIPA (Tudor IT Service Management Process Assessment) [3] methodology which is compliant with ISO/IEC 15504-2 [4]. Section 7 examines how TIPA uses the requirements contained in ISO/IEC 15504-2 as a guide for its transformation process for the development of Process Reference Models (PRM) and Process Assessment Models (PAM). Section 8 examines how an assessment model could be developed using the TIPA transformation process which could be used to assess medical IT networks against IEC 80001-1. Finally, section 9 contains the conclusions of this research and plans to progress this work further.

\section{IEC 80001-1 Overview}

\subsection{What is IEC 80001?}

Medical Devices are designed and validated in order to ensure that they are safe for their intended use. Increasingly Medical Devices are being produced which are intended to be incorporated into Hospital IT networks. Medical devices are integrated into heterogeneous networks that include other medical devices and other IT 
components creating a new system in which the medical device has not been validated. New hazards may emerge that are directly related to the interaction of the networked components that were not considered when the device was being designed and validated [5]. Each Healthcare Delivery Organisation (HDO) will approach the integration of a medical device into an IT network in a different way. There are a number of potential problems which are associated with the incorporation of medical devices into hospital IT networks as follows [1]:

- "Lack of consideration for risk from use of IT networks during evaluation of clinical risk."

- "Lack of support from manufacturers of medical devices for the incorporation of their products into IT networks, (e.g. the unavailability or inadequacy of information provided by the manufacturer to the operator of the IT networks)."

- "Incorrect operation or degraded performance (e.g. incompatibility or improper configuration) resulting from combining medical devices and other equipment on the same IT networks."

- "Incorrect operation resulting from combining medical device software and other software applications (e.g. open email systems or computer games) in the same IT networks."

- "Lack of security controls on many medical devices."

- "The conflict between the need for strict change control of medical devices and the need for rapid response to the threat of cyber-attack."

The goal of IEC 80001-1 is to prevent patient harm. The meaning of harm as defined in IEC 80001-1 is extended to address 3 areas - Safety, Effectiveness and (Data \& System) Security - therefore covering in addition to physical injury or damage to the health of a patient, any reduction in the effectiveness of the device (in its ability to provide its intended result for the patient) and any breach of data and system security (any degradation of the confidentiality, integrity and availability of information assets) [5] .

IEC 80001-1 covers the entire life cycle of the Medical IT network. The standard incorporates the principle that a risk management process should be implemented when creating a medical device network or when removing, maintaining, or changing/modifying equipment on a medical device network. The risk based approach is based on the risk based approach outlined in ISO 14971 [6] which is used by medical devices manufacturers but extends the definition of harm to include reduction in effectiveness and breach of security

\subsection{Roles \& Responsibilities}

IEC 80001-1 is addressed to Responsible Organisations (defined within the standard as an entity responsible for the use and maintenance of a Medical IT network), Medical Device Manufacturers and to providers of other information technology. This part of the standard outlines the specific roles, responsibilities and activities (of these groups) with regard to risk management of the incorporation of medical devices into IT networks to address the safety, effectiveness and data \& system security. 
The IEC 80001-1 standard acknowledges that the overall responsibility for the Medical IT network belongs to the HDO. Medical device networks are becoming increasingly complex and the clinical users within the HDO may not recognise the risks to patients presented by the incorporation of medical devices onto the IT networks. The HDO must focus on gaining the support and assistance of internal functions and the suppliers of the components and products that will form part of the network in order to gain an understanding of the interactions within the network that present the greatest risk. With the assistance of these parties, the HDO must seek to identify factors over which it has the most control and focus on preventing or mitigating possible hazards based on these factors [5]. The top management of the HDO must establish a risk management policy for the incorporation of medical devices and must appoint a medical IT network risk manager who is appropriately skilled and qualified to administer the policy and who must maintain the medical IT network risk management file. The medical IT risk management file must contain all documentary information (including supplier documentation) as to support the risk management activities of the Medical IT network.

The IEC 80001-1 standard also requires that for a device to be connected to a Medical IT network, the manufacturer of the device must make supporting documents available to the HDO. The documentation must contain instructions for implementing the connection of the device to the medical IT network and should include the purpose of the devices connection to the network, the required characteristics, configuration and technical specification of the network to which the device is being connected, the intended information flow between the medical device, the Medical IT network and any other devices on the network and a list of the hazardous situations that may arise due to the failure of the IT network to meet the characteristics required by the medical device in order to support the connection to the IT network [1] . Should the information provided by the manufacturer be insufficient as to allow the HDO to carry out risk management activities, the HDO must request any additional information required from the medical device manufacturer.

\section{Developing Process Models for assessing against IEC 80001-1}

ISO/IEC 15504 is an international standard for process assessment that is widely adopted in the software engineering community. ISO/IEC 15504-2 [4] (ISO/IEC 15504 is also known as SPICE - Software Process Improvement \& Capability dEtermination) defines a framework for assessing capability of processes. The SPICE framework is widely used in the safety critical domain in such initiatives as Automotive SPICE and Medi SPICE. For a 15504 compliant process assessment, ISO/IEC 15504-2 requires the use of a PAM which is formulated by reference to one or more external PRMs. It also defines the requirements that process models (PAMs and PRMs) must meet. The ISO/IEC 15504-2 requirements are discussed in detail in the next section.

In order to assess against IEC 80001-1, (in a manner that is compliant with ISO/IEC 15504-2) it is necessary that a PAM is developed. In order to develop this model, we examined how other process models have been developed in order to perform an assessment against similar standards. 
Annex D of IEC 80001-1 shows that there is a strong relationship between IEC 80001-1 and ISO/IEC 20000-1 \& 2 - Information Technology - Service Management. We discovered that ISO/IEC 15504-8 (Information Technology Process Assessment - Part 8: An exemplar process assessment model for IT Service Management) had also been developed to assess against ISO/IEC 20000-1. The ISO/IEC 15504 series does not provide guidance today on how to develop PAMs; we can use the TIPA transformation process instead. Within this paper we focus on the TIPA [3] process models and examine how they were developed by using the requirements of ISO/IEC 15504-2 as a framework to assess against ISO/IEC 20000-1 and ITIL [7] - Information Technology Infrastructure Library processes [8]. From this examination, we investigate how to apply the TIPA transformation process $^{1}$ to develop a PRM and a PAM for assessing against IEC 80001-1.

\section{Process Assessment and ISO/IEC 15504}

ISO/IEC 15504 provides a framework for the assessment of processes. ISO/IEC 15504-2 defines a measurement framework for process capability and defines the requirements for performing an assessment, building PRMs, PAMs and verifying conformity of process models and of process assessment. The standard looks at process assessment from the basis of a two dimensional model containing both a process dimension and a capability dimension. The process dimension is provided by reference to an external PRM which characterises processes in terms of their purpose and outcomes. The capability dimension is based on 6 capability levels. The achievement of these capability levels is based on the achievement of the associated process attributes. Further guidelines for process description are outlined in ISO/IEC TR 24774:2010 [9].

ISO/IEC 15504-5 [10] provides an exemplar PAM for software processes which can be used to perform an assessment conformant to ISO/IEC 15504-2. The PAM expands the PRM process definitions by including a set of process performance and process capability indicators. Process performance indicators are called base practices for each process. The PAM also defines a second set of indicators of process performance by associating work products with each process. Process capability indicators are generic practice, generic resource and generic work product.

There are two aspects within process assessment, process capability determination and process improvement. Process capability seeks to assess the current state of a set of processes against the defined capability levels and process attributes. A target capability level will have been determined in advance of the assessment. The results of the assessment are then analysed to determine the strengths, weaknesses, opportunities and threats in the process context and process improvement can be undertaken on this basis.

\footnotetext{
${ }^{1}$ We will use «TIPA transformation process » to make reference to the Transformation Process used to build the TIPA process models, as documented in [14].
} 


\section{ISO/IEC 20000 - Service Management Standards}

Service Management looks at a lifecycle approach to managing IT services to ensure that the services continually not only provide the required service to the customer but also provide value to the customer in terms of their business goals. ISO/IEC 20000-1 "requires an integrated process approach when the service provider plans, establishes, implements, operates, monitors, reviews, maintains and improves a service management system (SMS)” [2].

In order to achieve this integrated process approach, ISO/IEC 20000 advocates a "Plan, Do, Check, Act" approach which is described briefly as follows [2].

- "Plan: establishing, documenting and agreeing the SMS. The SMS includes the policies, objectives, plans andprocesses to fulfil the service requirements.'

- "Do: implementing and operating the SMS for the design, transition, delivery and improvement of the services."

- "Check: monitoring, measuring and reviewing the SMS and the services against the policies, objectives, plansand service requirements and reporting the results."

- "Act: taking actions to continually improve performance of the SMS and the services."

The most important aspect of the integrated methodology and "Plan, Do, Check, Act" approach are detailed in the standard as follows:

- "understanding and fulfilling the service requirements to achieve customer satisfaction"

- " "establishing the policy and objectives for service management"

- "designing and delivering services based on the SMS that add value for the customer"

- "monitoring, measuring and reviewing performance of the SMS and the services"

- "continually improving the SMS and the services based on objective measurements"

ISO/IEC 20000-2 [11] describes the best practices for Service Management within the scope of ISO/IEC 20000-1. This part of the standard provides guidance on the 5 process categories indentified in Part 1 of the standards namely Service Delivery Processes, Control Processes, Release Processes, Resolution Processes and Relationship Processes. Part 4 [12] of the standard (ISO/IEC 20000-4) provides the PRM for IT Service Management based on the requirements of ISO/IEC 20000-1. It should be noted that the TIPA methodology provides an assessment framework to assess against either ISO/IEC 20000-4 or against the processes contained within the widely used Service Management best practice library - ITIL [7]. ITIL was developed in the United Kingdom at the end of the 1980's. Now owned by the Cabinet Office, ITIL has become the world wide 'de facto' standard for IT Service Management [3]. ITIL v3 [7] has been organised into 5 publications which cover - Service Strategy, 
Service Design, Service Transition, Service Operation and Continual Service Improvement.

ISO/IEC 20000-4 and ITIL are very similar standards - both dealing with a lifecycle approach to Service Management. There is a close relationship between ITILv3 and ISO/IEC 20000 to the extent that ISO/IEC 20000 has become known as the "ITIL standard". With the publication of ISO/IEC 20000-1:2011, there have been steps taken to ensure that ISO/IEC 20000 is more closely aligned with ITIL v3 [13]. Given the alignment between ITILv3 and the ISO/IEC 20000 series, the TIPA methodology is equally effective at assessing ISO/IEC 20000 and ITILv3 processes [3]. The following section looks at the relationship between IEC 80001-1 and ISO/IEC 20000.

\section{IEC 80001-1 - Relationship with ISO/IEC 20000}

\subsection{Service Management in the lifecycle of a Medical Device Network.}

IEC 80001-1 addresses risk management throughout the life cycle of the Medical IT network. The monitoring of Medical IT networks during operation may reveal the need for a change to the device. Due to the stringent regulation of medical devices, HDOs must follow strictly formal approaches and procedures directly involving the medical device manufacturer when making changes to or performing maintenance activities on medical devices. This establishes an on-going relationship between the manufacturer of the device and the HDO which continues throughout the lifecycle of the Medical IT network. This relationship is constrained by the need on the part of the HDO to change a medical device and the need for the medical device manufacturer to ensure that the changed device continues to be validated. It must be understood by the medical device manufacturer and the HDO that these opposing constraints impact service management [1] . Lifecycle risk management must be performed in a way that allows the HDO to support effective healthcare delivery. In this context, the principles of Service Management outlined in ISO/IEC 20000 have been reviewed to determine their ability to meet the requirements of IEC 80001-1. It should however be noted that compliance with ISO/IEC 20000-1 does not equate to compliance with IEC 80001-1.

\subsection{Relationship between IEC 80001-1 and ISO/IEC 20000}

IEC 80001-1 applies lifecycle risk management to Medical IT networks. IEC 80001-1 states that "Lifecycle risk management in a medical IT-network needs to be done in the context of the specific operating conditions required to support effective healthcare delivery" [1]. Due to the common lifecycle approach, the concepts of Service Management as described in ISO/IEC 20000-1 and ISO/IEC 20000-2 [11] have been examined for their ability to meet the requirements outlined in IEC 800011. The relationship between IEC 80001-1 and ISO/IEC 20000 is described in Annex $\mathrm{D}$ of IEC 80001-1. This annex provides a simple overview of the relationship between IEC 80001-1 and ISO/IEC 20000-2 to aid in the investigation of service 
strategies that could address the service needs of a medical IT-network. Figure 1 provides an overview of the areas described in Annex D of IEC 80001-1.

The top portion of the diagram shows areas identified within the standards wherein the terminology may differ but the underlying role, document or the processes are similar e.g. what is referred to in IEC 80001-1 as "Risk Management Process" appears as "Security Risk Assessment Practices" within ISO/IEC 20000, but the underlying process areas are the same.

The lower portion of the diagram shows areas within the lifecycle that are common to both standards. For example IEC 80001-1: Figure 2 shows an overview of the "Overview of life cycle of medical IT-networks including risk management"[1] which shows "Change - Release Management" (including the risk assessment process) through the "Go Live" implementation of the change and to finally "Live Environment Risk Management" (including monitoring of the change and event management). These processes are common to ISO/IEC 20000-1 as shown in Figure D.1 of IEC 80001-1[1] which lists the ISO/IEC 20000-1 processes as Control Processes (including Change Management), Release Processes (including Release Management) and Resolution Processes (including Problem \& Incident Management). The additional processes identified in Figure D.1 including Service Delivery Processes and Relationship processes (including Supplier Management) are also common to both standards.

Given the relationship between the standards, our research has focused on assessment frameworks that are currently available to assess against ISO/IEC 20000. The following section 7 of this paper examines how the TIPA process models were developed to assess against ISO/IEC 20000.

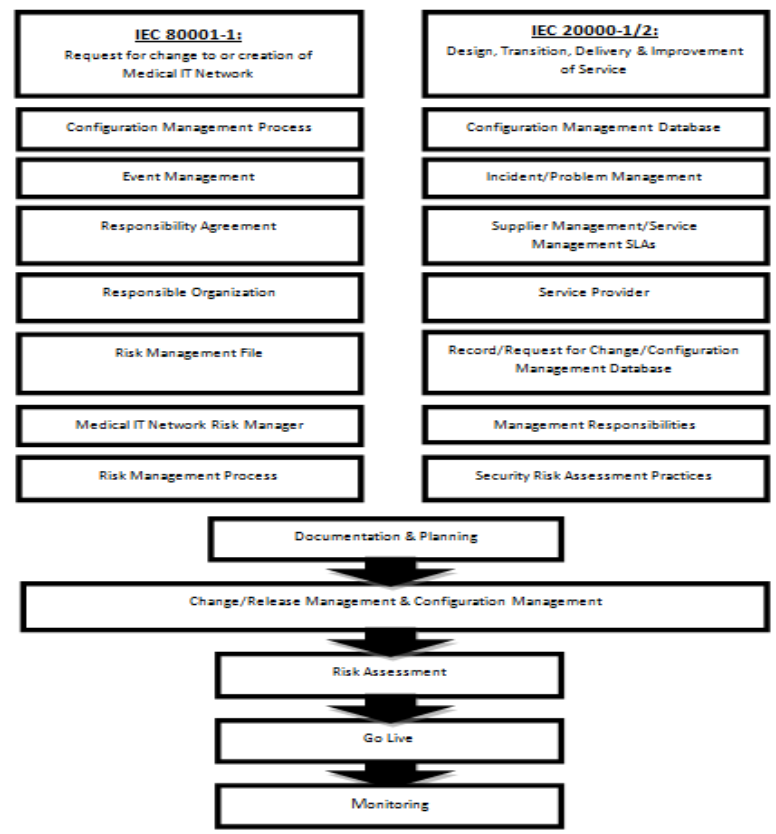

Fig. 1. Provides an overview of the relationship between IEC 80001-1 and ISO/IEC 200001:2011 and ISO/IEC 20000-2:2005 showing aligned teminology and common processes. 


\section{TIPA Methodology}

This section of the paper examines how the TIPA methodology has combined the requirements expressed in ISO/IEC 15504 and used these requirements to build process models to assess against ISO/IEC 20000 and ITIL. As per ISO15504-2, in order to perform a process assessment, a PAM must be developed with reference to one or more PRMs. The TIPA methodology advocates the use of a transformation process, based on goal-oriented requirements engineering techniques, in order to develop the required PRMs and PAMs [14]. An explanation of the transformation process is given in section 7.1.

The development of the TIPA PAM for ISO/IEC 20000-1 was based on the existing PRM described in ISO/IEC 20000-4. Barafort et al. [14] focussed on developing the PAM using the transformation process outlined within the next section. The TIPA PAM for ISO/IEC 20000 (which was developed using the TIPA methodology) was one of the inputs to ISO/IEC 15504-8 that is further developed in JTC1 ISO/IEC SC7 [15]. ISO/IEC 15504-8 "provides an example of an IT Service Management Process Assessment Model (PAM) for use in performing a conformant assessment in accordance with the requirements of ISO/IEC 15504-2.” [15].

In developing the PAM for assessment against ITIL, no PRM existed so a PRM based on ITILv3 and a PAM were developed using the same transformation process. The TIPA transformation process can be used for the development of both PRMs and PAMs. A PAM is formed on the basis of one or more PRMs and the addition of a measurement framework. As the TIPA transformation process can be used for the development of both PRMs and PAMs, this methodology could also be used to develop the PRM and PAM for IEC 80001-1.

\subsection{TIPA Transformation Process Overview}

As examined in section 6, ISO/IEC 15504 provides a detailed description of the process assessment approach and provides an exemplar PAM in ISO/IEC 15504-5 [10]. ISO/IEC TR 24774:2010 provides guidelines for the formulation of process descriptive elements [9]. However, Barafort et al. identified that a gap exists in that there is no guidance to support the transformation from the input (domain requirements) to the output (process model) [14]. The CRP Henri Tudor has produced a PAM for ISO/IEC 15504 compliant assessment against both ISO/IEC 20000 and ITIL. The TIPA transformation process is as follows [14]:

1. Identify elementary requirements in a collection of requirements.

2. Organise and structure the requirements.

3. Identify common purposes upon those requirements and organise them towards domain goals.

4. Identify and factorise outcomes from the common purposes and attach them to the related goals.

5. Group activities together under a practice and attach it to the related outcomes.

6. Allocate each practice to a specific capability level. 
7. Phrase outcomes and process purpose. (Apply ISO/IEC TR 24774 guidelines)

8. Phrase the Base Practices attached to the Outcomes. (Apply ISO/IEC TR 24774 guidelines)

9. Determine Work Products among the inputs and outputs of the practices.

Based on the transformation process above TIPA PAMs have been developed to assess against ISO/IEC 20000 and ITIL.

Assessment against ISO/IEC 20000 focuses on the processes within 2 process categories: Primary Processes and Organisational Processes. The Primary Processes consist of the following process groups: Service Delivery Process Group, Relationship Process Group, Resolution Process Group, Control Process Group and Release Process Group. The Organisational Processes consists of one process group: Planning and Implementing Service Process Group. Assessment against ITIL focuses on the processes within 2 process category groups of version 2: Service Support Group and Service Delivery Group.

TIPA applies the ISO/IEC 15504 assessment method to IT Service Management as a means to verify the maturity of the process. In applying the requirements expressed in ISO/IEC 15504-2 to build process models for ISO/IEC 20000-1 and ITIL, TIPA provides a means to assess the maturity of IT Service Management processes.

\section{How the TIPA Transformation Process may be applied to IEC 80001-1}

Given the common areas in terms of lifecycle risk management and service management processes between ISO/IEC 20000 and IEC 80001-1 as defined in Section D of the IEC 80001-1 standard, it is clear that a Process Assessment Model could be built using the TIPA transformation process that would provide a framework against which to assess medical IT networks. Using the TIPA transformation process will ensure that the resultant PAM will meet the requirements of process assessment models as outlined in ISO/IEC 15504-2.

The diagram below shows how a PRM and PAM could be developed for assessment against IEC 80001-1 based on the TIPA transformation process. 


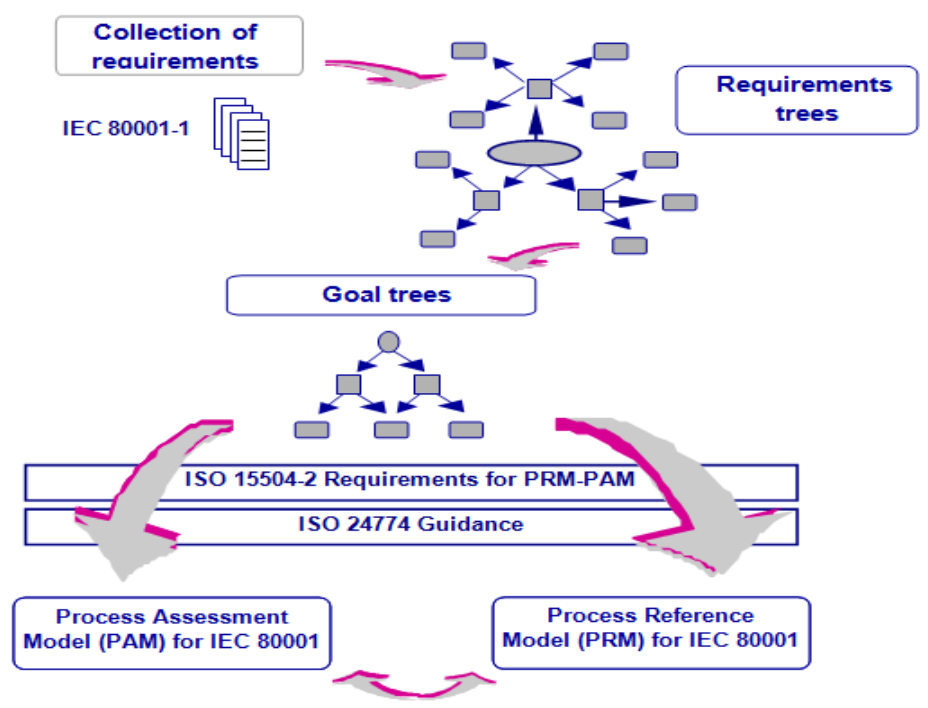

Fig. 2. Shows how the TIPA transformation process may be used to create a process reference model and process assessment to assess medical IT networks against IEC 80001-1.

\section{Conclusions \& Future Work}

Traditionally medical devices have functioned as stand-alone products which often meant that there was a need for information from devices to be transcribed or transferred to systems holding patient medical records. Placing a medical device onto the network and transferring the information directly removes the burden of transferring this information and removes the risk of human error in transferring the information impacting on patient care. However, the introduction of medical devices onto non-proprietary networks may risk compromising the safety of the device, the effectiveness of the device in its ability to produce the intended result for the patient or may result in a breach of data and system integrity. IEC 80001-1 seeks to mitigate these risks by applying a lifecycle risk management approach to the establishment or modification of medical device networks.

In order to perform an assessment (which is compliant with ISO/IEC 15504-2) on a Medical IT networks against IEC 80001-1, a Process Assessment Model is required. IEC 80001-1 Annex D shows the relationship between ISO/IEC 20000 and IEC 80001-1. TIPA has been developed as a means of assessing Service Management processes against ISO/IEC 20000-1. Given this overlap in processes and the common lifecycle approach it is proposed that a Process Assessment Model could be developed, using the TIPA transformation process, to assess against IEC 80001-1. This approach would also ensure compliance with ISO/IEC 15504-2.

Future research will focus on the formulation of a Process Reference Model and Process Assessment Model based on the TIPA transformation process. An iterative approach will be followed. Each process will be validated by industry experts and amended according to the consensus. Once the PRM and PAM have been fully developed, the model will then be validated through trials. 


\section{Acknowledgements}

This research is supported by the Science Foundation Ireland (SFI) Stokes Lectureship Programme, grant number 07/SK/I1299, the SFI Principal Investigator Programme, grant number 08/IN.1/I2030 (the funding of this project was awarded by Science Foundation Ireland under a co-funding initiative by the Irish Government and European Regional Development Fund), and supported in part by Lero - the Irish Software Engineering Research Centre (http://www.lero.ie) grant 10/CE/I1855.

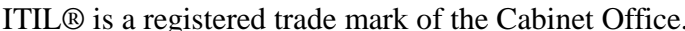

TIPA $®$ is a Registered Trade Mark of the CRP Henri Tudor

\section{References}

1. IEC, IEC 80001-1 - Application of Risk Management for IT-Networks incorporating Medical Devices - Part 1: Roles, responsibilities and activities. 2010, International Electrotechnical Commission: Geneva, Switzerland.

2. ISO/IEC, ISO/IEC 20000-1:2011 - Information technology - Service management Part 1: Service management system requirements. 2011: Geneva, Switzerland.

3. Barafort, B., V. Betry, S. Cortina, M. Picard, M. St Jean, A. Renault, O. Valdés, and P.R.C.H. Tudor, ITSM Process Assessment Supporting ITIL : Using TIPA to Assess and Improve your Processes with ISO 15504 and Prepare for ISO 20000 Certification. Best Practice. Vol. 217. 2009, Zaltbommel, Netherlands: Van Haren.

4. ISO/IEC, ISO/IEC 15504-2 - Software engineering - Process assessment - Part 2: Performing an assessment. 2003: Geneva, Switzerland.

5. Cooper, T., Y. David, and S. Eagles, Getting Started with IEC 80001: Essential Information for Healthcare Providers Managing Medical IT-Networks. 2011: Association for the Advancement of Medical Instrumentation. 76.

6. ISO, ISO 14971:2007 - Medical Devices - Application of Risk to Medical Devices. 2007, International Organisation for Standardization: Geneva, Switzerland.

7. Cartlidge, A., A. Hanna, C. Rudd, I. Macfarlane, J. Windebank, and S. Rance, An introductory Overview of ITILv3 - A high-level overview of the IT INFRASTRUCTURE LIBRARY. 2007, The UK Chapter of the itSMF.

8. Barafort, B., B. Di Renzo, and O. Merlan, Benefits Resulting from the Combined Use of ISO/IEC 15504 with the Information Technology Infrastructure Library (ITIL) Product Focused Software Process Improvement, M. Oivo and S. Komi-Sirviö, Editors. 2002, Springer Berlin / Heidelberg. p. 314-325.

9. ISO/IEC, ISO/IEC TR 24774:2010 - Systems and software engineering - Life cycle management - Guidelines for process description. 2010: Geneva, Switzerland.

10. ISO/IEC, ISO/IEC 15504-5 - Information technology - Process Assessment - Part 5: An exemplar Process Assessment Model. 2006: Geneva, Switzerland.

11. ISO/IEC, ISO/IEC 20000-2:2005 - Information technology -- Service management -- Part 2: Code of Practice. 2005: Geneva, Switzerland.

12. ISO/IEC, ISO/IEC TR 20000-4:2010 - Information technology - Service management Part 4: Process reference model. 2010: Geneva, Switzerland.

13. Dugmore, J. and S. Taylor (2008) ITILv3 and ISO/IEC 20000 - Alignment White Paper March 2008. Best Management Practice for IT Service Management.

14. Béatrix Barafort, Alain Renault, Michel Picard, and S. Cortina, A transformation process for building PRMs and PAMs based on a collection of requirements - Example with ISO/IEC 20000, in SPICE 2008: Nuremberg, Germany.

15. ISO/IEC, ISO/IEC PDTR 15504-8 - Information technology -- Process assessment -- Part 8: An exemplar process assessment model for IT service management. 2011: Geneva, Switzerland. 